[5] S. Mahapatra, V. Vaish, C. Wasshuber, K. Banerjee, and A. M. Ionescu, "Analytical modeling of single electron transistor (SET) for hybrid CMOS-SET analog IC design," IEEE Trans. Electron. Devices, vol. 51, no. 11 , pp. 1772-1782, Nov. 2004.

[6] H. Grabert and M. H. Devoret, Single Charge Tunneling: Coulomb Blockade Phenomena in Nanostructures. New York: Plenum, 1992.

[7] I. Koren, Computer Arithmetic Algorithms, 2nd ed. Natick, MA: A. K. Peters, 2002.

[8] M. M. Dastjerdi, A. A. Kusha, and M. Pedram, "BZFAD: A low power low area multiplier based on shift and add architecture," IEEE Trans. Very Large Scale Integr. (VLSI) Syst., vol. 17, no. 2, pp. 302-306, Feb. 2009.

[9] S. S. Dan and S. Mahapatra, "Impact of energy quantization on the performance of current-biased SET circuits," IEEE Trans. Electron. Devices, vol. 56, no. 8, pp. 1562-1566, Aug. 2009.

[10] M. Goossens, J. Ritskes, C. Verhoeven, and A. van Roermund, "Learning single electron tunneling neural nets," in Proc. ProRISC Workshop Circuits, Syst. Signal Process., 1997, pp. 179-186.

\section{Fast Transient Low-Dropout Voltage Regulator With Hybrid Dynamic Biasing Technique for SoC Application}

Chia-Min Chen, Student Member, IEEE, Tung-Wei Tsai, and Chung-Chih Hung, Senior Member, IEEE

\begin{abstract}
This brief presents a low-dropout (LDO) voltage regulator without output capacitors that achieves fast transient responses by hybrid dynamic biasing. The hybrid dynamic biasing in the proposed transient improvement circuit is activated through capacitive coupling. The proposed circuit senses the LDO regulator output change so as to increase the bias current instantly. The proposed circuit was applied to an LDO regulator without output capacitors implemented in standard $0.35-\mu \mathrm{m}$ CMOS technology. The device consumes only $25 \mu \mathrm{A}$ of quiescent current with a dropout voltage of $180 \mathrm{mV}$. The proposed circuit reduces the output voltage spike of the LDO regulator to $80 \mathrm{mV}$ when the output current is changed from 0 to $100 \mathrm{~mA}$. The output voltage spike is reduced to $20 \mathrm{mV}$ when the supply voltage varies between 1.3 and $2.3 \mathrm{~V}$ with a load current of $100 \mathrm{~mA}$.
\end{abstract}

Index Terms-Capacitive coupling, hybrid dynamic biasing, low-dropout regulator, transient response, voltage spike.

\section{INTRODUCTION}

Nowadays, on-chip power management units have been extensively developed, implying there are multiple power domains in the system-on-chip (SoC) design. The power management system scales down the supply voltage by low-dropout (LDO) to power many circuit blocks. Transient response time is an important dynamic specification in LDO designs because the voltage spike affects the overall performance. For the LDO loop bandwidth, it is necessary to control the locations of the loop's poles and zeros in smallsignal analysis [1]-[3]. Increasing the loop bandwidth can improve small-signal performance at low and moderate frequencies. If the design focuses on the large-signal behavior, typical approaches are to increase the bias current to achieve a high slew rate, or to use large capacitors to reduce the undershoot and overshoot of the output

Manuscript received November 22, 2011; revised July 6, 2012; accepted August 31, 2012. Date of publication October 9, 2012; date of current version August 2, 2013. This work was supported in part by the National Science Council and National Chip Implementation Center.

The authors are with the Institute of Communications Engineering, National Chiao Tung University, Hsinchu 300, Taiwan (e-mail: jeremy. cm97g@g2.nctu.edu.tw; twtsai.cm96g@g2.nctu.edu.tw; cchung@mail.nctu. edu.tw).

Color versions of one or more of the figures in this paper are available online at http://ieeexplore.ieee.org.

Digital Object Identifier 10.1109/TVLSI.2012.2217766

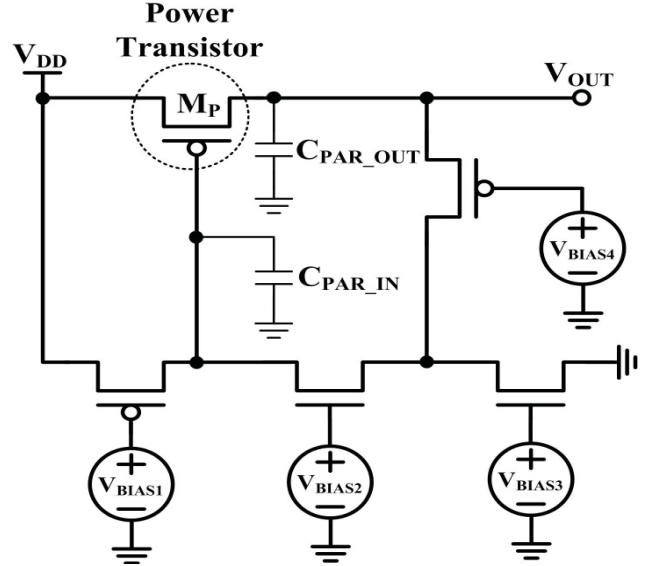

Fig. 1. LDO structure without output capacitors.

voltage $\left(V_{\text {OUT }}\right)$ [4], [5]. In SoC applications [6]-[9], to reduce the undershoot or overshoot of the output voltage, the use of a large output capacitor will require an extra pin, which is therefore not preferred. To effectively increase the slew rate instantly seems to be a better approach to cope with output voltage spikes.

There are many ways to solve the voltage spike problem. One method is to use a constant bias current to increase the slew rate, where the bias current is not dependent on the output current. Besides, a previous design incorporates a $600-\mathrm{pF}$ on-chip capacitor to reduce the output voltage ripple [10]. This method does not meet the powersaving and area-limited requirements of SoC designs.

The other method is the push-pull biasing method [11]. More bias current will be used at the transient instant only if the output current changes. The error amplifier uses a push-pull output stage to increase the current for charging and discharging the gate capacitance of the power transistor $\left(C_{\text {PAR_IN }}\right)$ during the transient instant. A differential-input common-gate amplifier activates the push-pull output stage. However, the fast-changing voltage spike cannot be detected effectively because the differential common-gate amplifier has limited bandwidth. This approach is also not suitable for low output voltages.

Another method is to raise the bias current based on the significance of the output current [12], [13]. For circuits to react to a change in the output voltage quickly, an adaptive bias current should maintain a minimum value in the steady state.

In this brief, an LDO with hybrid dynamic biasing is proposed to improve the output voltage transient speed under abrupt changes of the output current. Through capacitive coupling, the fast transient circuit senses transient changes in voltage at the LDO output and instantly increases the bias current. The quiescent current is only $25 \mu \mathrm{A}$.

The rest of this brief is organized as follows. Section II presents the LDO architecture and operation principle of the proposed fast transient circuit through hybrid dynamic biasing. Section III shows experimental results. The final section addresses the conclusion of this brief.

\section{LDO ARCHITECTURE}

\section{A. LDO Architecture Without the Output Capacitor}

Fig. 1 shows an LDO structure without the output capacitor [10]. Fig. 2 illustrates the large-signal responses of this structure. In Fig. 2(a), when the output voltage $\left(V_{\text {OUT }}\right)$ suddenly drops, it instantly decreases the $V_{\mathrm{SG}}$ of transistor $\mathrm{M}_{1}$ and turns it off. To provide the same bias current (I $I_{\text {BIAS }}$ ), $C_{\text {PAR_IN }}$ itself releases the current. In this 


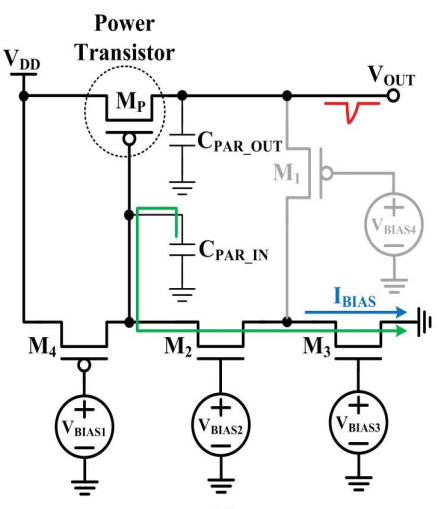

(a)

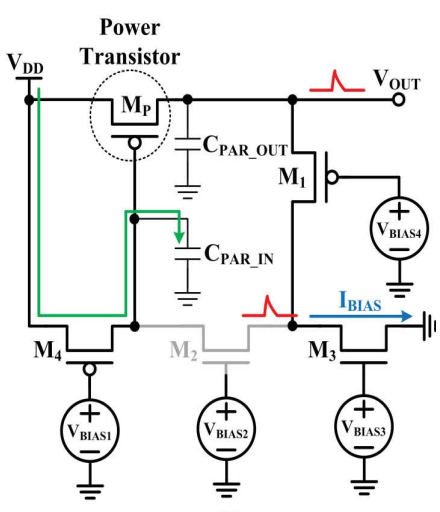

(b)
Fig. 2. Large-signal responses of the LDO structure without an output capacitor. (a) Undershoot. (b) Overshoot.

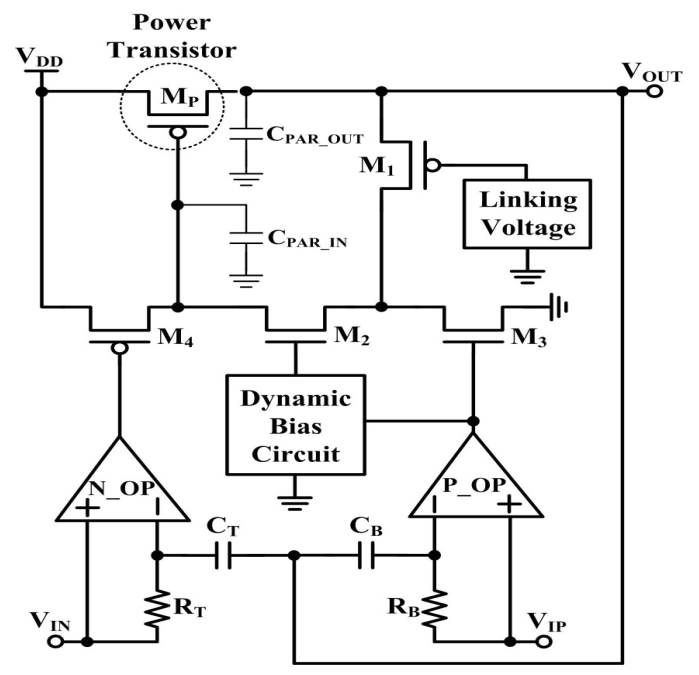

Fig. 3. LDO structure with the proposed fast transient circuit through hybrid dynamic biasing.

way, the current flowing through transistor MP increases when the $V_{\mathrm{SG}}$ of transistor MP increases. This current pulls $V_{\mathrm{OUT}}$ back to the original voltage. Fig. 2(b) shows that, when $V_{\text {OUT }}$ rises suddenly, it increases the drain voltage of transistor $\mathrm{M}_{1}$ and the source voltage of transistor $\mathrm{M}_{2}$. Thus, a decrease in the $V_{\mathrm{GS}}$ of transistor $\mathrm{M}_{2}$ turns off transistor $\mathrm{M}_{2}$. As a result, all current flowing through transistor $\mathrm{M}_{4}$ is charged to $C_{\text {PAR_IN }}$. This phenomenon increases the gate voltage of transistor MP and decreases the $V_{\mathrm{SG}}$ of transistor MP. This effectively reduces the current through the transistor MP. To maintain the same $I_{\mathrm{BIAS}}, V_{\mathrm{OUT}}$ is pulled down to keep the same $I_{\mathrm{BIAS}}$ current, thereby returning to the original voltage. The power transistor has a large size and generates a large parasitic gate capacitance. Owing to the large parasitic capacitance and the high impedance at this node, the gate of the power transistor forms a dominant pole. The other nodes are all of low impedance. Therefore, in the negative feedback loop, there only appears one pole within loop bandwidth, so the LDO does not require an output capacitor, which is therefore suitable for SoC applications.

\section{B. Structure and Operation Principles of the Proposed Fast Transient Circuit}

Fig. 3 illustrates the proposed architecture. The most critical feature of the structure is the dynamic enhancement of the slew rate. When the output voltage changes dramatically, the bias current immediately changes to improve the slew rate and reduce the LDO undershoot and overshoot. Figs. 4 and 5 analyze the large-signal performance

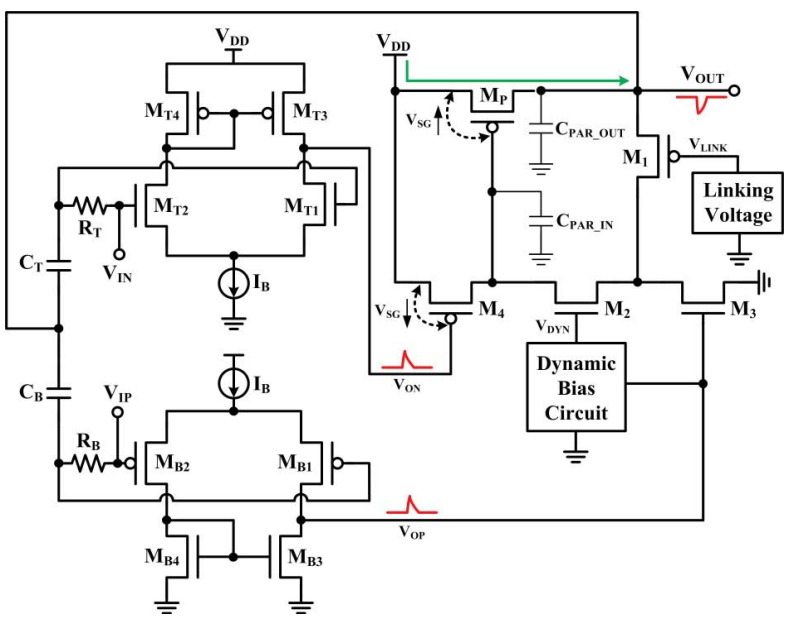

Fig. 4. Operation of the proposed circuit (undershoot).

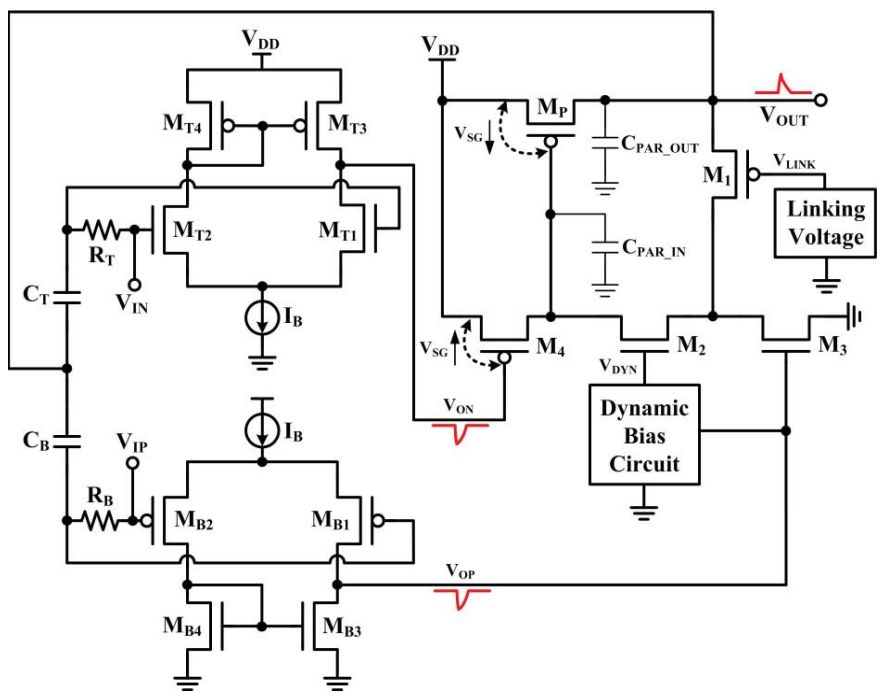

Fig. 5. Operation of the proposed circuit (overshoot).

of undershoot and overshoot, respectively. The voltage gain of the two op amps is $A_{v}=-g_{m 1}\left(r_{o 1} / / r_{o 3}\right)$. The gain of the proposed circuit is larger than that of the circuit in [8], enabling it to amplify the detected transient signals more efficiently to enhance the transient driving capabilities and slew rates of transistors $\mathrm{M}_{4}$ and $\mathrm{M}_{3}$. Thus, the gate charge of power transistor $M_{P}$ can be promptly sourced or sunk, i.e., the power transistor can respond efficiently. Each op amp consumes only $3.5 \mu \mathrm{A}$.

In addition to the capacitive coupling circuit, we also propose a dynamic bias circuit. The dynamic bias circuit can speed up the discharge of transistor $\mathrm{M}_{2}$ according to the transient output of the capacitive coupling circuit, thereby reducing the response time of the power transistor. In contrast, the circuit in [8] uses a fixed bias voltage, which limits the response time of the power transistor, resulting in a larger variation of the output voltage unless a larger bias current has been used. The inclusion of the dynamic bias circuit enables the power transistor to respond quickly to an abrupt increase in load, thereby reducing the variation of the output voltage and expediting the recovery of output voltage. The detailed operation of the dynamic bias circuit is given in Section II-D.

Fig. 4 shows that, when $V_{\text {OUT }}$ suddenly drops, the capacitive coupling of $V_{\text {OUT }}$ causes the gate voltage of transistors $\mathbf{M}_{B 1}$ and $\mathrm{M}_{T 1}$ to drop. This, in turn, increases the output voltage of two differential amplifiers, decreasing the $V_{\mathrm{SG}}$ of transistor $\mathrm{M}_{4}$ and 


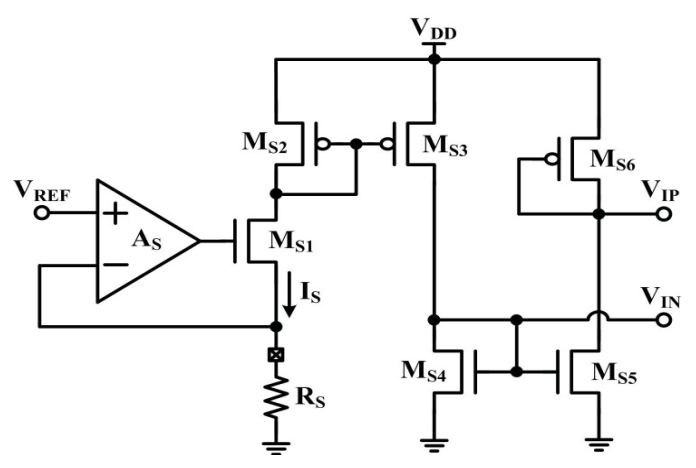

Fig. 6. Bias generation circuit.

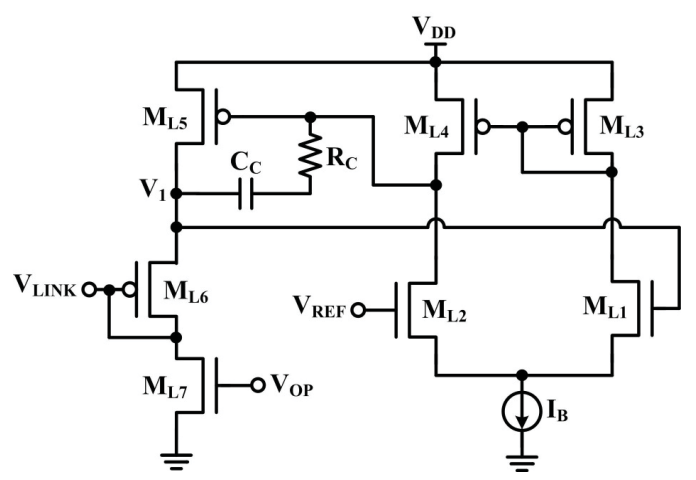

Fig. 7. Linking voltage generation circuit.

increasing the $V_{\mathrm{GS}}$ of transistor $\mathrm{M}_{3}$. The current flowing through transistor $\mathrm{M}_{3}$ becomes higher. As the $V_{\mathrm{SG}}$ of transistor $\mathrm{M}_{4}$ decreases, the current flowing through transistor $\mathrm{M}_{4}$ decreases. This instantly removes current from $C_{\text {PAR_IN }}$, causing the gate voltage of transistor $\mathrm{M}_{P}$ to drop quickly. When the $V_{\mathrm{SG}}$ of transistor $\mathrm{M}_{P}$ increases, it increases the current flowing through transistor $\mathrm{M}_{P}$. This, in turn, charges the output parasitic capacitance $\left(C_{\text {PAR_OUT }}\right)$ and pulls $V_{\text {OUT }}$ back to the original steady state. In Fig. 5 , when $V_{\text {OUT }}$ suddenly rises, the capacitive coupling of $V_{\text {OUT }}$ causes the gate voltage of transistors $\mathrm{M}_{B 1}$ and $\mathrm{M}_{T 1}$ to rise. This decreases the output voltage of two differential amplifiers, increasing the $V_{\mathrm{SG}}$ of transistor $\mathrm{M}_{4}$ and decreasing the $V_{\mathrm{GS}}$ of transistor $\mathrm{M}_{3}$. In this case, the current flowing through transistor $\mathrm{M}_{3}$ decreases, and the current flowing through transistor $\mathrm{M}_{4}$ increases. Thus, some current flowing through transistor $\mathrm{M}_{4}$ charges $C_{\mathrm{PAR}} \mathrm{IN}$, which increases the gate voltage of transistor MP. In this way, the $V_{\mathrm{SG}}$ of transistor MP decreases, which decreases the current flowing through transistor MP. Thus, $C_{\text {PAR_OUT }}$ discharges, making $V_{\text {OUT }}$ to pull down and return to the original steady voltage. In order to reduce the overshoot, increasing the gate voltage of the power transistor $\mathrm{M}_{P}$ is more effective than discharging LDO output by transistor $\mathrm{M}_{1}$.

The bias generation circuit for $V_{\mathrm{IP}}$ and $V_{\mathrm{IN}}$ of Figs. 4 and 5 is shown in Fig. 6. The op amps in the proposed LDO regulator require additional bias voltages ( $V_{\mathrm{IP}}$ and $\left.V_{\mathrm{IN}}\right)$, while the circuit of [8] also requires two additional bias voltages.

\section{Proposed Linking Voltage Generation Circuit (LVGC)}

Fig. 7 shows that $V_{\mathrm{REF}}$ locking $V_{1}$ makes $V_{1}=V_{\mathrm{REF}}$ by connecting op amp in the unity-gain feedback configuration. The op amp provides one stage of high gain and one stage of low gain. The closed-loop gain is $A_{f} \approx g_{\mathrm{mL} 1}\left(r_{\mathrm{oL} 4} / / r_{\mathrm{oL} 2}\right)\left(2 g_{\mathrm{mL} 5} / g_{\mathrm{mL} 6}\right)=$ $46 \mathrm{~dB}$. The gain, which is larger than that of [8], is sufficient to force $V_{1}$ to nearly equal $V_{\mathrm{REF}}$. The LVGC is designed to make the currents flowing through transistor $\mathrm{M}_{L 6}$ and $\mathrm{M}_{1}$ to be the same. The size of transistor $M_{L 6}$ equals the size of transistor $M_{1}$,

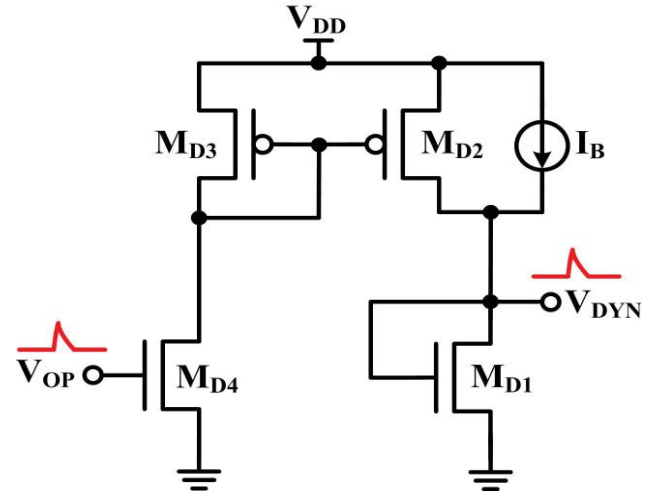

Fig. 8. Dynamic bias circuit.

ensuring that $V_{\mathrm{SG}(\mathrm{ML} 6)}=V_{\mathrm{SG}(\mathrm{M} 1)}$. Therefore, the output voltage $V_{\text {OUT }}=V_{1}=V_{\text {REF }}$. Meanwhile, the bias current is designed as $I_{\mathrm{ML7}}=I_{\mathrm{ML} 6}=I_{M 1}$ by selecting the size of transistor $\mathrm{M}_{3}$ to be twice that of $\mathrm{M}_{L 7}$.

\section{Proposed Dynamic Bias Circuit}

The design principle of the dynamic bias circuit is to make $V_{\mathrm{DYN}}$ change with $V_{\mathrm{OP}}$, as shown in Fig. 8 . The primary purpose of the dynamic bias circuit is to momentarily change the bias current of the feedback loop of the main circuit when voltage spikes appear at the LDO output in order to quickly charge or discharge gate parasitic capacitance $\left(C_{\mathrm{PAR} \_\mathrm{IN}}\right)$ of the power transistor. A sudden drop in $V_{\mathrm{OUT}}$ increases $V_{\mathrm{OP}}$, which decreases the drain voltage of transistor $\mathrm{M}_{D 4}$. A decrease in the drain and gate voltage of transistor $\mathrm{M}_{D 3}$ decreases the gate voltage of transistor $\mathrm{M}_{\mathrm{D} 2}$. Because the $V_{\mathrm{SG}}$ of transistor $\mathrm{M}_{D 2}$ is larger, the current flowing through it becomes larger as well, which causes $V_{\mathrm{DYN}}$ to increase instantly. A drop in $V_{\text {OUT }}$ causes an increase in $V_{\text {DYN }}$. A sudden drop at the gate of power transistor $\left(\mathrm{M}_{P}\right)$ increases the $V_{\mathrm{SG}}$ of the power transistor as well as the current flowing through it. This enables a more rapid pull-up of $V_{\text {OUT }}$, returning it to a steady voltage. The addition of the dynamic bias circuit makes the whole circuit respond even faster with better performance.

\section{E. Enhancement of Transient Responses}

In Fig. 3, the high-pass network comprises resistor $R_{T}\left(R_{B}\right)$ and capacitor $C_{T}\left(C_{B}\right)$. The values of both $C_{T}$ and $C_{B}$ are $2 \mathrm{pF}$ and the values of both $R_{T}$ and $R_{B}$ are $400 \mathrm{k} \Omega$. Thus, the corner frequency of the high-pass network is $199 \mathrm{kHz}$. When the load current of the LDO regulator is $100 \mathrm{~mA}$, the crossover frequency of the main feedback loop is $500 \mathrm{kHz}$. The corner frequency of the high-pass network must be designed to be less than the crossover frequency of the LDO feedback loop. $V_{\text {OUT }}$ needs to vary at least $5 \mathrm{mV}$ within $5 \mu \mathrm{s}$, so the fast transient circuit and the dynamic bias circuit can be efficiently triggered to respond to and reduce the output spikes.

Fig. 9 shows the measurement results of the load transient responses when $V_{\mathrm{DD}}=1.3 \mathrm{~V}, V_{\mathrm{OUT}}=1.1 \mathrm{~V}$, and $I_{\mathrm{OUT}}=$ 1-100 mA. There are three parts in Fig. 9. The top one is the load transient response of the LDO with the proposed circuit, and the middle one is that of the LDO without the proposed circuit. The bottom one shows that IOUT changes between 1 and $100 \mathrm{~mA}$. Both the rise and fall time of the load current range from 1 to $100 \mathrm{~mA}$ is $200 \mathrm{~ns}$. The proposed dynamic hybrid biasing improves the undershoot of the output voltage from 480 to $117 \mathrm{mV}$, while the recovery time is improved from 6 to $1.4 \mu \mathrm{s}$. The overshoot is improved from 173 to $28 \mathrm{mV}$. The proposed approach effectively extends the bandwidth of the feedback loop, and ensures high-speed transient response to signal change. 


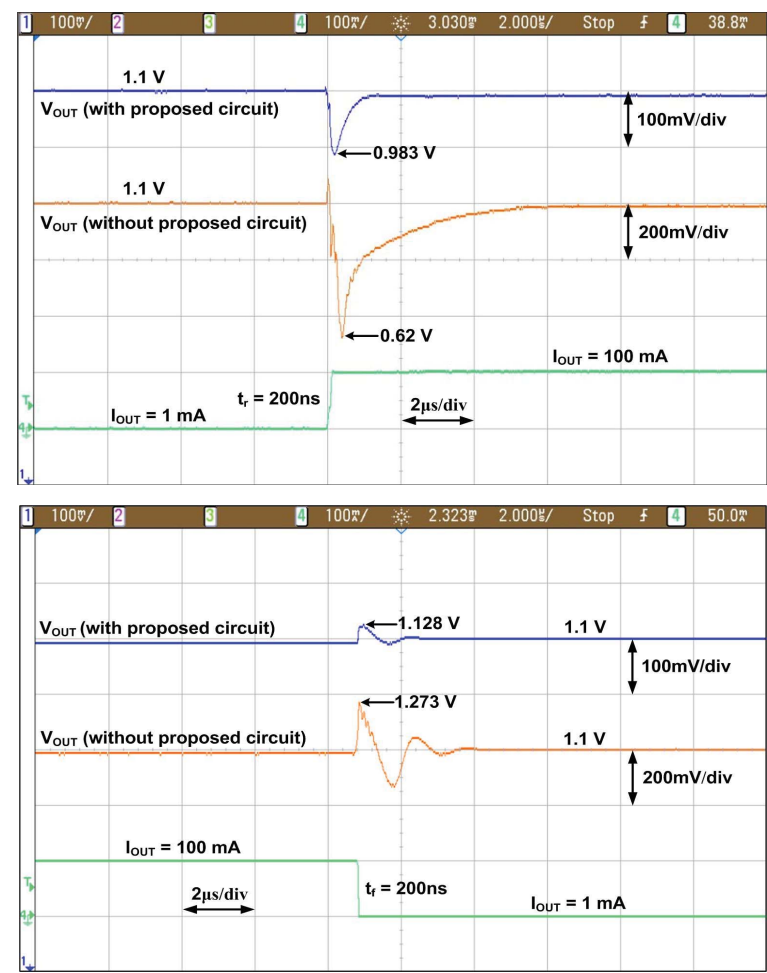

Fig. 9. Comparison of two LDO structures with and without the proposed fast transient circuit, at $V_{\mathrm{DD}}=1.3 \mathrm{~V}, V_{\mathrm{OUT}}=1.1 \mathrm{~V}, I_{\mathrm{OUT}}(\mathrm{MAX})=$ $100 \mathrm{~mA}, I_{\mathrm{OUT}}(\mathrm{MIN})=1 \mathrm{~mA}$.

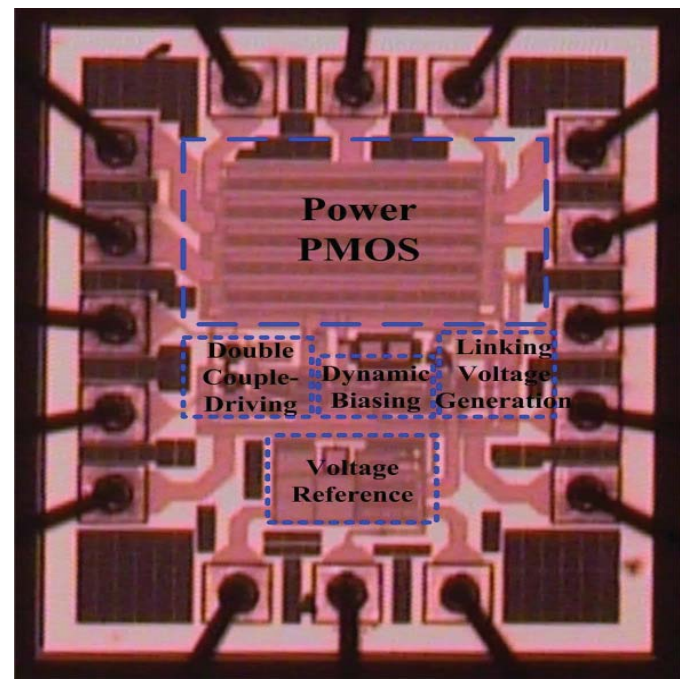

Fig. 10. Die photograph of the proposed LDO voltage regulator.

\section{EXPERIMENTAL RESULTS}

The proposed LDO regulator was fabricated using the $0.35-\mu \mathrm{m}$ mixed-signal CMOS $2 \mathrm{P} 4 \mathrm{M}$ process. The design of our regulator uses only $3.3 \mathrm{~V}$ MOS transistors; therefore, the maximum operation voltage is $3.3 \mathrm{~V}$. Fig. 10 shows the die photograph of the prototype voltage regulator. Fig. 11 shows the input-output voltage characteristics of LDO regulator at $I_{\mathrm{OUT}}=100 \mathrm{~mA}$, the dropout region, and line regulation performance. The size of the power transistor is $26000 \mu \mathrm{m} / 0.35 \mu \mathrm{m}$ to provide high output current. The dropout voltage is $180 \mathrm{mV}$. Fig. 12 shows the measured line transient response. The supply voltage switches between 1.3 and $2.3 \mathrm{~V}$ with $100 \mathrm{~mA}$ output current, and voltage spike is $20 \mathrm{mV}$. Fig. 13 shows the measured load transient response without an off-chip output capacitor. The output current varies from 0 to

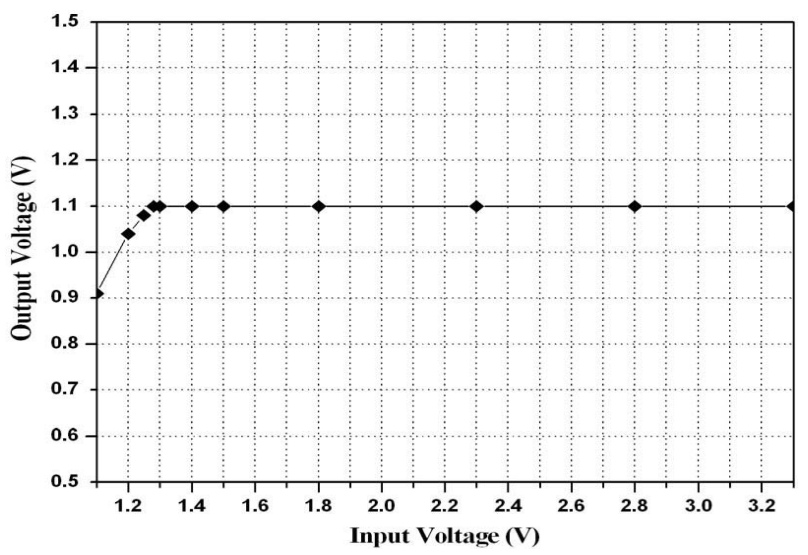

Fig. 11. Input-output characteristics of the regulated voltage.

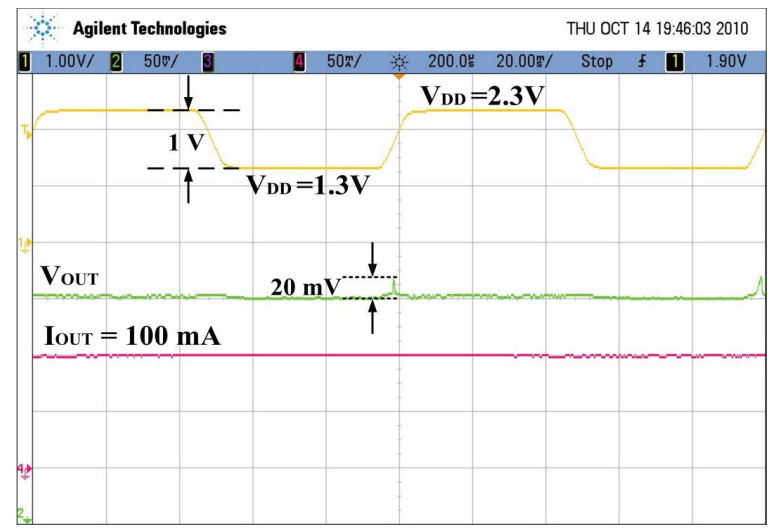

Fig. 12. Line transient measurement result of the proposed LDO voltage regulator without an off-chip output capacitor.

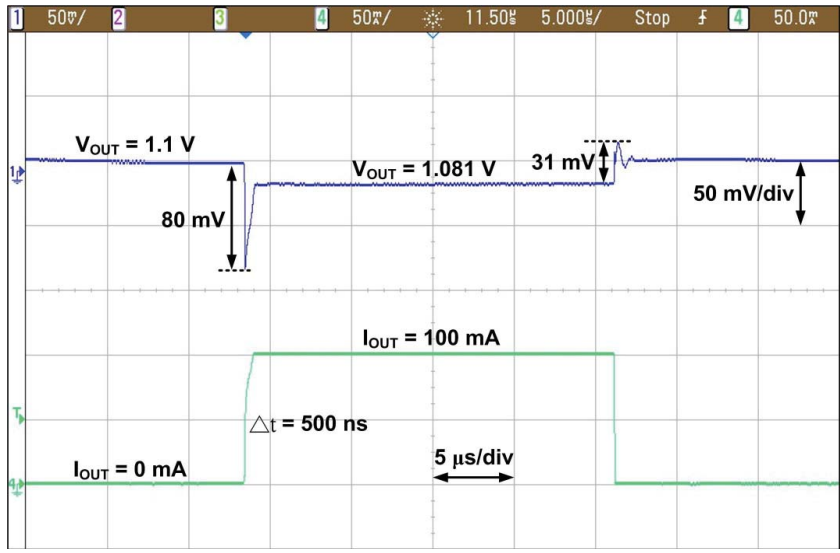

Fig. 13. Load transient measurement result of the proposed LDO voltage regulator without an off-chip output capacitor.

$100 \mathrm{~mA}$ at $1.3 \mathrm{~V}$ supply voltage, while the rise time and fall time of the load current are $5 \mathrm{~ns} / \mathrm{mA}$. These measurement results show that the load transient voltage spike is only $80 \mathrm{mV}$.

The quiescent current of $25 \mu \mathrm{A}$ was measured with a load current $I_{\mathrm{OUT}}=100 \mathrm{~mA}$. All bias current flows into the ground; therefore, we simply measure the current flowing into the ground pin to obtain the quiescent current. The current efficiency was obtained from (1). Fig. 14 shows the current efficiency as a function of load current

$$
\eta_{I}=\frac{I_{\mathrm{OUT}}}{I_{\mathrm{GND}}+I_{\mathrm{OUT}}}=\frac{100 \mathrm{~mA}}{25 \mu \mathrm{A}+100 \mathrm{~mA}}=99.97 \% .
$$

Performance comparison between some previously reported LDOs and the proposed LDO is summarized in Table I. The figure of merit 
TABLE I

Performance Comparison With Previously Reported LDOs

\begin{tabular}{|c|c|c|c|c|c|}
\hline & {$[2]$} & {$[4]$} & {$[8]$} & {$[10]$} & This Brief \\
\hline Technology $(\mu \mathrm{m})$ & 0.5 & 0.35 & 0.35 & 0.09 & 0.35 \\
\hline Supply Voltage (V) & $1.4-4.2$ & 2.0 & $0.95-1.4$ & 1.2 & $1.28-3.3$ \\
\hline $\mathrm{I}_{\mathrm{OUT}, \mathrm{MAX}}(\mathrm{mA})$ & 100 & 200 & 100 & 100 & 100 \\
\hline $\mathrm{V}_{\mathrm{OUT}}(\mathrm{V})$ & 1.21 & 1.8 & 1.2 & 0.9 & 1.1 \\
\hline $\mathrm{I}_{q}$ & $45 \mu \mathrm{A}$ & $20 \mu \mathrm{A}$ & $43 \mu \mathrm{A}$ & $6000 \mu \mathrm{A}$ & $25 \mu \mathrm{A}$ \\
\hline $\mathrm{V}_{\text {dropout }}(\mathrm{V})$ & 0.2 & 0.2 & 0.2 & 0.3 & 0.18 \\
\hline$\Delta \mathrm{V}_{\mathrm{OUT}}(\mathrm{mV})$ & $\begin{array}{c}120 \\
\left(\Delta \mathrm{I}_{\mathrm{OUT}}=100 \mathrm{~mA}\right)\end{array}$ & $\begin{array}{c}54 \\
\Delta \mathrm{I}_{\mathrm{OUT}}=200 \mathrm{~mA}\end{array}$ & $\begin{array}{c}70 \\
\left(\Delta \mathrm{I}_{\mathrm{OUT}}=99 \mathrm{~mA}\right)\end{array}$ & $\begin{array}{c}90 \\
\left(\Delta \mathrm{I}_{\mathrm{OUT}}=100 \mathrm{~mA}\right)\end{array}$ & $\begin{array}{c}80 \\
\left(\Delta \mathrm{I}_{\mathrm{OUT}}=100 \mathrm{~mA}\right)\end{array}$ \\
\hline Current efficiency & $99.95 \%$ & $98.8 \%$ & $99.95 \%$ & $94.3 \%$ & $99.97 \%$ \\
\hline Current Capacitor & $100 \mathrm{nF}$ & $1 \mu \mathrm{F}$ & $0-100 \mathrm{pF}$ & $600 \mathrm{pF}$ & $0-100 \mathrm{pF}$ \\
\hline $\mathrm{FOM}_{1}(\mathrm{ps})$ & 59 & 27 & 0.03 & 32 & 0.02 \\
\hline $\mathrm{T}_{\mathrm{R} \text { (measure) }}(\mu \mathrm{s})$ & 2.5 & 15.4 & 3 & 0.00054 & 0.75 \\
\hline $\mathrm{FOM}_{2}(\mathrm{~ns})$ & 1.125 & 1.54 & 1.29 & 0.032 & 0.187 \\
\hline Active area & $0.263 \mathrm{~mm}^{2}$ & $0.264 \mathrm{~mm}^{2}$ & $0.155 \mathrm{~mm}^{2}$ & $0.098 \mathrm{~mm}^{2}$ & $0.126 \mathrm{~mm}^{2}$ \\
\hline
\end{tabular}

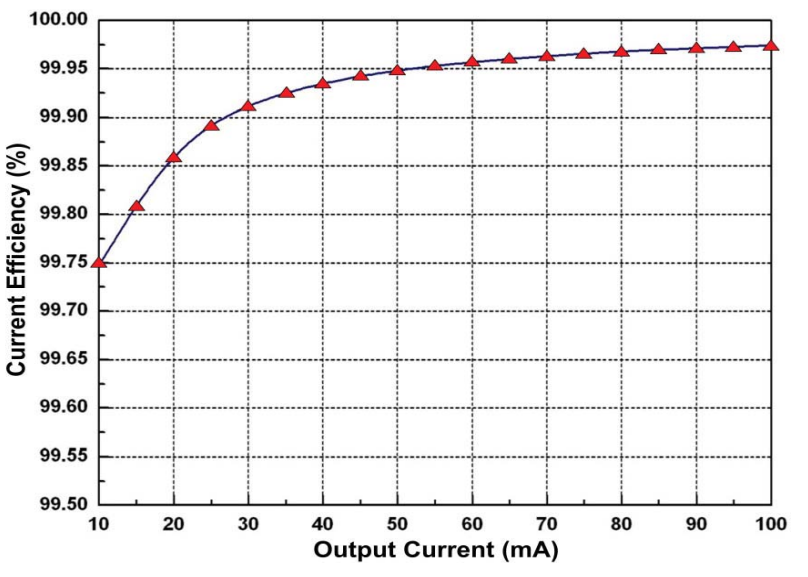

Fig. 14. Current efficiency as a function of load current.

(FOM), given in (2) [10], is adopted in Table I

$$
\mathrm{FOM}_{1}=T_{R} \frac{I_{q}}{I_{\mathrm{OUT}, \mathrm{MAX}}}=\frac{C_{\mathrm{OUT}} \cdot \Delta V_{\mathrm{OUT}}}{I_{\mathrm{OUT}, \mathrm{MAX}}} \frac{I_{q}}{I_{\mathrm{OUT}, \mathrm{MAX}}} .
$$

To show an FOM directly obtained from the measurement data, a measured response time $\left(T_{\mathrm{R} \text { (measure) }}\right)$ from load transient responses is taken into account. Another FOM is defined as

$$
F O M_{2}=T_{\mathrm{R}(\text { measure) }} \frac{I_{q}}{I_{\mathrm{OUT}, \mathrm{MAX}}} .
$$

Although our $\mathrm{FOM}_{2}$ is larger than that of [10], the latter actually has an on-chip output capacitance of $600 \mathrm{pF}$, which has effectively reduced the measured response time. Therefore, to compare the performance with [10], $\mathrm{FOM}_{1}$ seems to be a fair index. In addition, our measurement condition for the load transient response ranges from 0 to $100 \mathrm{~mA}$, while the measurement condition in [8] ranges from 1 to $100 \mathrm{~mA}$. The output impedance changes abruptly for load currents ranging from 0 to $100 \mathrm{~mA}$; however, the output impedance does not change a great deal for load currents ranging from 1 to $100 \mathrm{~mA}$. In other words, the measurement conditions for load transient response are far stricter in this case than in [8]. Therefore, the proposed LDO regulator is superior both in static and transient characteristics to the LDO regulator in [8]. By using 0.35- $\mu \mathrm{m} \mathrm{CMOS}$ technology, the chip area of the proposed design is smaller than that in both [4] and [8].

\section{CONCLUSION}

This brief presented an LDO voltage regulator without the output capacitor that achieves fast transient responses by hybrid dynamic biasing. The dynamic biasing in the proposed fast transient circuit is activated through capacitive coupling. The fast transient through hybrid dynamic biasing circuit senses the LDO output change so as to increase the bias current instantly. The output voltage spike of the LDO with the proposed circuit decreases to $80 \mathrm{mV}$ when the output current changes from 0 to $100 \mathrm{~mA}$ and $20 \mathrm{mV}$ when a supply voltage of $1 \mathrm{~V}$ input step is applied to the circuit.

The proposed LDO regulator is stable for output current in the complete range of $0-100 \mathrm{~mA}$ and does not require any off-chip output capacitor. The proposed circuit improves both the overshoot and the undershoot of the LDO regulator. The FOM of this LDO compares favorably with that of other published designs.

\section{REFERENCES}

[1] G. A. Rincon-Mora, "Active capacitor multiplier in Miller-compensated circuits," IEEE J. Solid-State Circuits, vol. 35, no. 1, pp. 26-32, Jan. 2000.

[2] A. Garimella, M. W. Rashid, and P. M. Furth, "Reverse nested miller compensation using current buffers in a three-stage LDO," IEEE Trans. Circuits Syst. II, Exp. Briefs, vol. 57, no. 4, pp. 250-254, Apr. 2010.

[3] Y.-H. Lin, K.-L. Zheng, and K.-H. Chen, "Smooth pole tracking technique by power MOSFET array in low-dropout regulators," IEEE Trans. Power Electron., vol. 23, no. 5, pp. 2421-2427, Sep. 2008.

[4] M. Al-Shyoukh, H. Lee, and R. Perez, "A transient-enhanced lowquiescent current low-dropout regulator with buffer impedance attenuation," IEEE J. Solid-State Circuits, vol. 42, no. 8, pp. 1732-1742, Aug. 2007.

[5] C. K. Chava and J. Silva-Martinez, "A frequency compensation scheme for LDO voltage regulators," IEEE Trans. Circuits Syst. I, Reg. Papers, vol. 51, no. 6, pp. 1041-1050, Jun. 2004.

[6] Y.-H. Lee and K.-H. Chen, "A $65 \mathrm{~nm}$ sub-1 V multi-stage low-dropout (LDO) regulator design for SoC systems," in Proc. IEEE Int. Midwest Symp. Circuits Syst. Conf., Aug. 2010, pp. 584-587.

[7] R. J. Milliken, J. Silva-Martinez, and E. Sanchez-Sinencio, "Full onchip CMOS low-dropout voltage regulator," IEEE Trans. Circuits Syst. I, Reg. Papers, vol. 54, no. 9, pp. 1879-1890, Sep. 2007.

[8] P. Y. Or and K. N. Leung, "An output-capacitorless low-dropout regulator with direct voltage-spike detection," IEEE J. Solid-State Circuits, vol. 45 , no. 2, pp. 458-466, Feb. 2010.

[9] C.-M. Chen and C.-C. Hung, "A capacitor-free CMOS low-dropout voltage regulator," in Proc. IEEE Int. Symp. Circuits Syst. Conf., May 2009, pp. 2525-2528.

[10] P. Hazucha, T. Karnik, B. A. Bradley, C. Parsons, D. Finan, and S. Borkar, "Area-efficient linear regulator with ultrafast load regulation," IEEE J. Solid-State Circuits, vol. 40, no. 4, pp. 933-940, Apr. 2005.

[11] T. Y. Man, P. K. T. Mok, and M. Chan, "A high slew-rate push-pull output amplifier for low-quiescent current low-dropout regulators with transient-response improvement," IEEE Trans. Circuits Syst. II, Exp. Briefs, vol. 54, no. 9, pp. 755-759, Sep. 2007. 
[12] G. A. Rincon-Mora and P. E. Allen, "A low-voltage, low quiescent current, low drop-out regulator," IEEE J. Solid-State Circuits, vol. 33, no. 1, pp. 36-44, Jan. 1998.

[13] W.-J. Huang, S.-H. Lu, and S.-I. Liu, "A capacitor-free CMOS low dropout regulator with slew rate enhancement," in Proc. Int. Symp. VLSI Design, Autom. Test, Apr. 2006, pp. 1-4.

\section{Digital Error Corrector for Phase Lead-Compensated Buck Converter in DVS Applications}

Shaowei Zhen, Xiaohui Zhu, Ping Luo, Yajuan He, and Bo Zhang

\begin{abstract}
Modern low-power system on a chip needs direct current converter with dynamic voltage scaling (DVS) ability for core power supply. The converter output should be accurate voltage across the full load current and voltage scaling range. An integrated buck converter for DVS application is proposed in this brief. Voltage mode phase lead compensation is implemented in the converter, with much smaller passive components than conventional type-III compensation. To improve accuracy, the output voltage error accompanied with load current and reference voltage caused by finite loop gain in analog control loop is corrected by the digital error corrector. The output voltage is compared by two comparators whose threshold voltage is about $10 \mathrm{mV}$ above and below the reference voltage, respectively. The duty cycle is slightly adjusted by finite state machine according to outputs of the two comparators. Experimental results show that the converter is well regulated over an output range of 0.7-1.8 V, with step voltage of $25 \mathrm{mV}$. When load current suddenly changes between 170 and $500 \mathrm{~mA}$, the overshoot and undershoot voltage are 32 and $50 \mathrm{mV}$, respectively. Load regulation is maintained about $1 \%$ throughout the full load range. The voltage error is within $\pm 10 \mathrm{mV}$ in the voltage scaling range.
\end{abstract}

Index Terms-Buck converter, digital error corrector, phase lead compensation, voltage mode control.

\section{INTRODUCTION}

Low-power design techniques have become increasingly important for modern system on chips (SoCs). The dynamic voltage and frequency scaling (DVFS) technique controls the supply voltage and operation frequency of each module in SoC in response to workload demands, leading to substantial power saving while maintaining system performance. Direct current (dc-dc) converter with dynamic voltage scaling (DVS) ability acts as a hardware platform in a typical DVFS system. It is one of the hot topics in current research [1]-[4]. Compared to traditional dc-dc converters, the converter in the DVFS system should have several extra characteristics [1], [2]. Efforts have been made to achieve fast voltage scaling response, by

Manuscript received January 24, 2012; revised August 7, 2012; accepted August 31, 2012. Date of publication October 9, 2012; date of current version August 2, 2013. This work was supported in part by the National S\&T Special Project of China under Grant 2009ZX01031-003-003, the Fundamental Research Funds for the Central Universities under Grant ZYGX2009J026, and the NLAIC Project under Grant 9140C0903091004.

S. Zhen and P. Luo are with the State Key Laboratory of Electronic Thin Films and Integrated Devices, Chengdu 610054, China (e-mail: swzhen@uestc.edu.cn; pingl@uestc.edu.cn).

$\mathrm{X}$. Zhu was with the University of Electronic Science and Technology of China, Chengdu 610054, China. He is now with Texas Instruments Semiconductor Technologies Company, Ltd., Shanghai 200030, China (e-mail: 326465632@qq.com).

Y. He and B. Zhang are with the University of Electronic Science and Technology of China, Chengdu 610054, China (e-mail: yjhe@uestc.edu.cn; zhangbo@uestc.edu.cn).

Color versions of one or more of the figures in this paper are available online at http://ieeexplore.ieee.org.

Digital Object Identifier 10.1109/TVLSI.2012.2217513 maximum current charging or hysteretic control [2]-[4]. Moreover, voltage accuracy over full load current and voltage scaling range is an important specification, because smaller design margin is allowed, leading to further power saving while eliminating any slack periods caused by egregious low power supply. Accurate output needs high gain error amplifier (EA) and power devices with low resistance. The type-III compensator is often implemented in buck converter for high loop gain, but large off-chip capacitors and resistors limit its implementation in integrated power converters. Though recent advances in research are successful for monolithically integrate type-III compensator by pseudotype-III compensation [5] or extending loop bandwidth [6], the passive components still occupy large area to generate low-frequency pole, or the loop bandwidth is limited [6]. In contrast, the phase lead compensator (PLC) stabilizes buck converter by only one high-frequency zero, so the area is tremendously reduced with comparable bandwidth with type-III compensation [7]. However, the primary drawback of the PLC is that the loop gain is severely curtailed. Thus, its application in DVFS systems is limited.

This brief details the design of a novel error correction method presented in [8]. The digital error corrector (DEC) is implemented in a phase lead-compensated buck converter. Extra digitally controlled offset voltage is introduced to cancel output error because of low loop gain and parasitical resistors. This brief is organized as follows. In Section II, the error correction scheme is proposed and the detailed circuit implementation is introduced. Section III shows and discusses experimental performance, and Section IV summarizes this brief.

\section{IMPLEMENTATION OF BUCK CONVERTER With DEC}

The simplified block diagram of the proposed buck converter with the proposed DEC is illustrated in Fig. 1. Output voltage $V_{\text {OUT }}$ is fed back to the PLC, and the operational transconductance amplifier (OTA) GM converts voltage error between $V_{\mathrm{REF}}$ and $V_{C}$ into differential current in $R_{1}$ and $R_{2}$, respectively. The differential current is compared with sawtooth current $I_{\mathrm{SAW}}$ by comparator Comp to generate pulse-width modulation (PWM) signal. $I_{\text {SAW }}$ and global clock signal CLK are provided by OSC. Power devices MP and $\mathrm{MN}$ are controlled and driven by RS latch and driver. There are two comparators designed accompanied with GM whose outputs are Comp_H and Comp_L, respectively. The threshold voltages to trigger Comp_H and Comp_L are defined as $V_{\text {OUT, MAX }}$ and $V_{\text {OUT, MIN }}$, respectively. The proposed DEC introduces digitally controlled current source at node $V_{1}$ to adjust duty cycle slightly according to Comp_H and Comp_L, until $V_{\text {OUT }}$ is between $V_{\text {OUT, MAX }}$ and

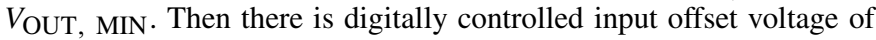
EA introduced by DEC. The original output voltage error due to low loop gain is compensated by the offset voltage. Thus the regulation and output accuracy are enhanced.

\section{A. Analog Control Loop}

The schematic of control blocks in Fig. 1, such as PLC, GM comparators, and $I_{\text {SAW }}$ generation, is shown in Fig. 2. PLC is realized by operational amplifier OP, resistors $R_{C 1}, R_{C 2}$, and capacitor $C_{C}$. GM is designed on the basis of symmetrical OTA. Voltage error between $V_{C}$ and $V_{\mathrm{REF}}$ is converted into current error in $R_{1}$ and $R_{2}$. $I_{\text {SAW }}$ generated by $I_{\text {SAW }}$ generation block flows out from node $V_{2}$ and output current of DEC flows from node $V_{1}$. The two comparators are current comparators, and $\mathrm{IB}_{1}$ and $\mathrm{IB}_{2}$ are designed to make $V_{\text {OUT, MAX about }} 10 \mathrm{mV}$ higher than $V_{\mathrm{REF}}$ and $V_{\text {OUT, MIN }}$ about $10 \mathrm{mV}$ lower. 